\title{
A System for Combined Visualization of EEG and Diffusion Tensor Imaging Tractography Data
}

\author{
Alexander Wiebel, Cornelius Müller, Christoph Garth, and Thomas R. Knösche
}

\begin{abstract}
In this paper we present an interactive system that integrates the visual analysis of nerve fiber pathway approximations from diffusion tensor imaging (DTI) with electroencephalography (EEG) data. The technique uses source reconstructions from EEG data to define certain regions of interest in the brain. These regions, in turn, are used to selectively display subsets of the approximated fiber pathways in the brain. The selected pathways highlight potential connections from activated areas to other parts of the brain and can thus help to understand networks on which most higher brain function relies. Users can explore the neuronal network and activity by navigating in an EEG curve view. The navigation is supported by optional mechanisms like snapping to time points with present reconstructed dipoles and visual cues highlighting such points. To the best of our knowledge, the presented combination of time navigation in EEG curves together with DTI pathway selection at the corresponding dipole positions is new and has not been described before. The presented methods are freely available in an open source system for visualization and analysis in neuroscience.
\end{abstract}

Key words: Diffusion tensor imaging, tractography, visualization, EEG, reconstructed dipoles, GUI

\footnotetext{
Alexander Wiebel

Zuse Institute Berlin (ZIB), Germany

Coburg University of Applied Sciences, Germany, e-mail: alexander.wiebel@hs-coburg.de

Cornelius Müller and Christoph Garth

Technische Universität Kaiserslautern, Germany

Thomas R. Knösche

Max Planck Institute for Cognitive and Brain Sciences, Leipzig, Germany
} 


\section{Introduction}

Neuroscience and neurology employ a large variety of measurement techniques to examine anatomy, function and pathologies of the human brain in vivo. Among them are electroencephalography (EEG), diffusion weighted magnetic resonance imaging (dwMRI), magnetoencephalography (MEG), and computed tomography (CT). Given the very complex structure of the brain, combining these techniques is crucial for gaining deeper insight into brain functions or diseases. In other words, multi-modal visualization and analysis tools are needed to provide neuroscientists and physicians the support they need for advanced research or diagnosis.

The work described in this paper is motivated by findings [28] that show correlations between epileptical networks measured using MEG or EEG, and connectivity of white matter fiber tracts. We present a system that enables the simultaneous inspection of EEG measurements and diffusion tensor imaging (DTI) data obtained from dwMRI measurements. In particular, a combination of dipole locations reconstructed from EEG (see e. g. [13]) and fiber pathways (see e. g. [2][10][16][18], Fig. 1) obtained from the DTI data is used for visualization, and an intuitive navigation for exploring the time dimension of the data is provided on the basis of simple curve plots of the measured EEG data. A user navigating in time triggers a search for the dipoles active at the current time. The positions of the found dipoles are then used to select fibers that run through activated brain areas represented by the dipoles (Fig. 2).

This way not only the brain activation related to a particular experimental or clinical condition can be visualized, but also the potential connections from the activated areas to other parts of the brain, i.e. the potentially activated networks. This is important as most higher brain function is thought to rely on widespread networks. Similar results can be achieved by a combination of fMRI and DTI [15][21][8]. However, in contrast to fMRI, EEG can achieve a very high resolution in time and thus is able to capture very fast current changes in the brain, which can occur within the order of milliseconds.

An illustration providing an overview of the overall pipeline of our approach is given in Fig. 3. It consists of the measurement, a preprocessing step and an interactive visualization and analysis loop. Throughout the paper we first describe how our approach is related to previous work (section 1.1), then we provide a brief introduction to the preprocessing (section 2) and finally introduce the new technique (section 3) as well as implementation details (section 4). The actually novel technique is integrated in the interactive visualization and analysis loop.

\subsection{Related Work}

The literature dealing with advanced EEG visualization is relatively sparse. Advanced in this context means showing more than the measured voltage curves (Fig. 2), a butterfly plot (Fig. 1) or frequency plots of the data. Exceptions are work 


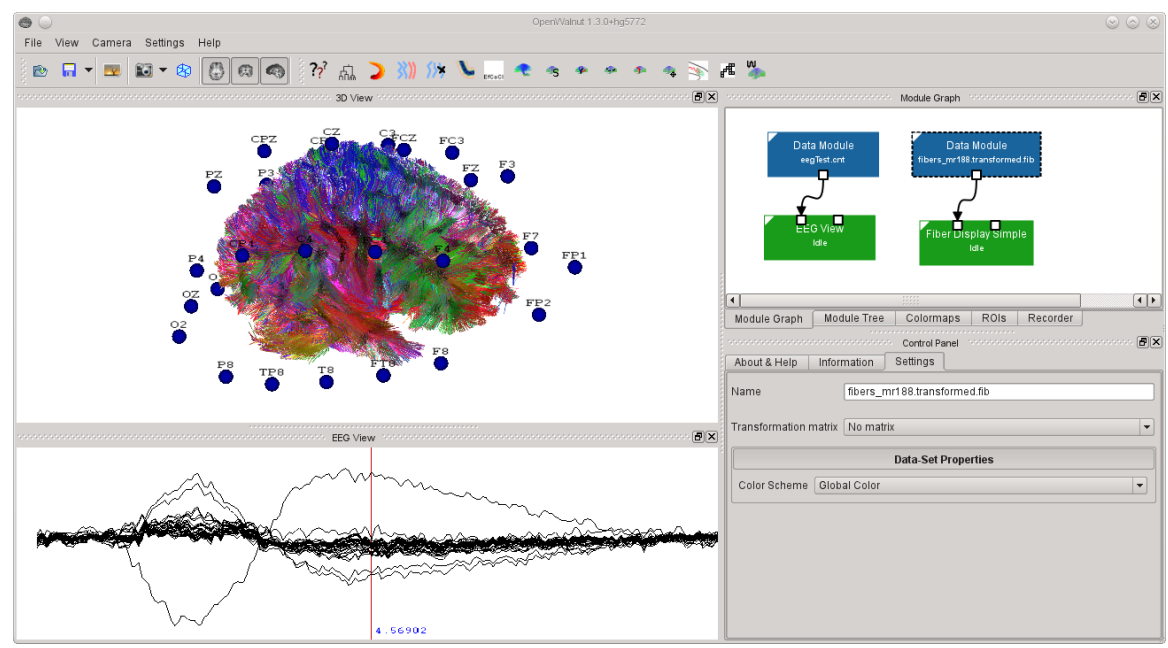

Fig. 1 DTI fibers tracts rendered in context of electrode positions in upper part of window and butterfly plot (details in section 3.2) of EEG recording in lower part.

on visualizing EEG recordings in 3D by Sourina et al. [23] and an information vi-

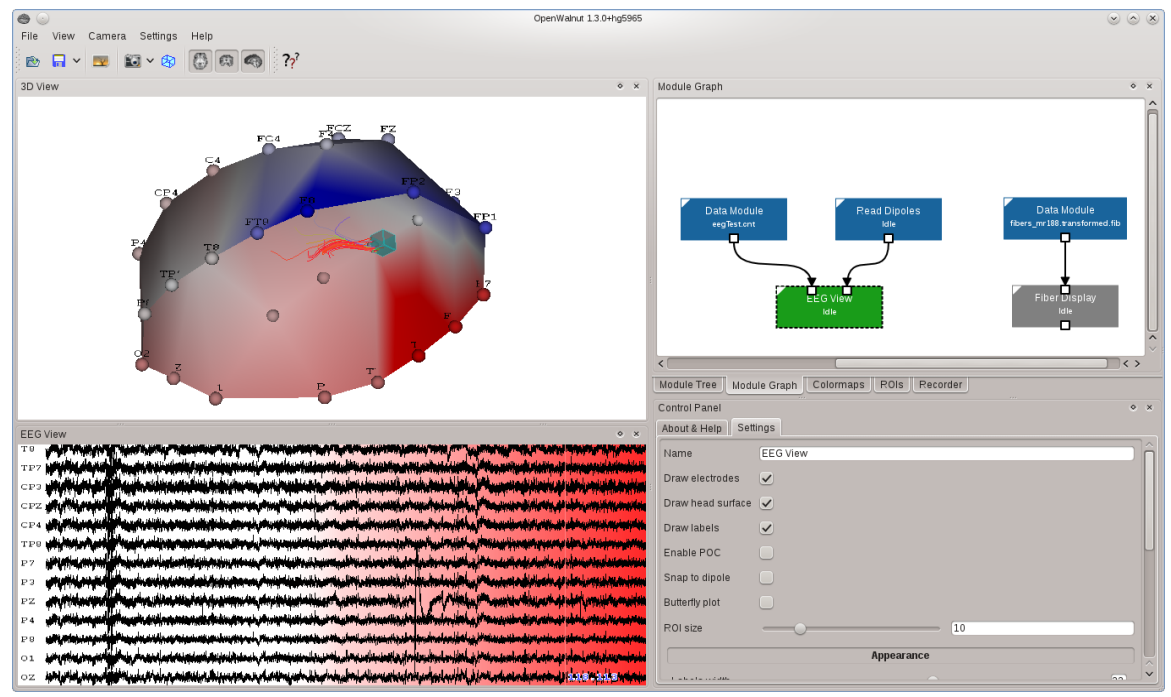

Fig. 2 DTI fibers tracts selected by the position of the reconstructed dipole $(10 \mathrm{~mm} \times 10 \mathrm{~mm} \times 10 \mathrm{~mm}$ ROI box) are rendered in context of head surface with voltage at the different electrodes mapped to color in the upper part of window. The lower part shows a common curve display of the EEG recording ("EEG View"). The "EEG View" widget is used for navigation in time (notice the vertical line marker, $t=118.113$ ). The color coding in the background indicates the presence of a loaded dipole. The color represents the magnitude of the dipole. 
sualization approach by ten Caat which he summarized in his doctoral thesis [25]. One of ten Caat's results is a method to visualize the coherence in EEG data as a network of interconnected functional units.

Some work regarding the analysis and visualization of sources reconstructed from EEG and MEG data (see Johnson et al. [11] and Wolters et al. [30]) has been performed. Wolters et al. [30] analyze result of source reconstructions from EEG data and their connection to data obtained from dwMRI. However, they do not employ the diffusion weighted data for approximating white matter fiber pathways for visualization, but to obtain conductivity tensors. The tensors are used to analyze the influence of the white matter conductivity on EEG/MEG field computation.

Discussions concerning the relation between functional MRI (fMRI) and EEG source reconstructions are quite common in the literature (see e.g. [27]). Also, the combination of fMRI and DTI-based fiber tracking to define and analyze white matter pathways is well established [15][21][8]. Such a combined analysis can be performed in many widely used software packages [9][26]. In contrast, the only work we found, that visualizes EEG data and fiber tracts, is the description of the NUTMEG system by Dalal et al. [4]. Their system, in conjunction with Xipy (also mentioned in the paper), shows white matter fiber tracts in conjunction with visualizations of functional maps obtained from source reconstruction. The system does not seem to provide an interactive navigation mechanism for inspecting reconstructions at different points in time and the original EEG recording curves are not visible to the user. It is furthermore unclear how the fiber tracts are selected.

In summary, there has been much work combining MRI and EEG (see also Tadel et al. [24]), few work dealing with DTI-based pathways together with EEG and no work allowing time navigation based on the display of voltage curves. This paper and the system presented in it aim at filling the gap left in the latter two areas.

\section{Preprocessing}

Acquiring the data during EEG and MRI measurements is only the very first step in a pipeline leading to the successful analysis of the subject's condition. The data needed for the effective utilization of the technique presented in this paper, have to be registered, tractography has to be applied to the DTI and dipoles have to be reconstructed from the EEG. As illustrated in Fig. 3, we consider these three parts of the pipeline as preprocessing steps and will detail two of them (tractography and reconstruction) in the following because the combination of their results lies in the heart of the presented technique. Naturally, the registration has to be as good as possible, but this is the case for most multi-modal visualization and analysis approaches and will thus not be discussed in the current context. 


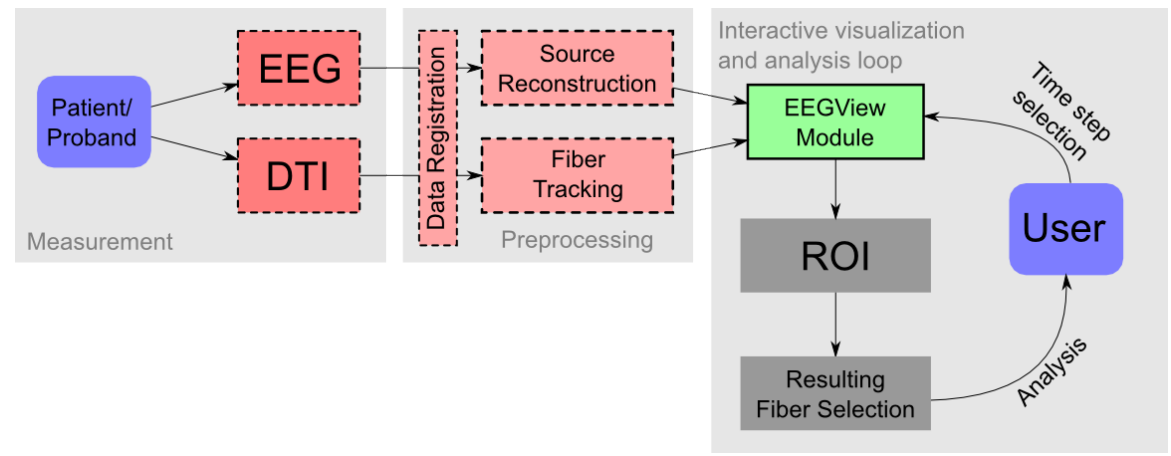

Fig. 3 Pipeline illustrating the overall approach. The central part of the system is integrated in the interactive visualization and analysis loop.

\subsection{DTI Tractography}

Approximated fiber pathways are one basis for the visualization and analysis of our approach. The data used for the fiber approximation in our example comes from a DTI measurement acquired with a 3-Tesla scanner Siemens $3 T$ Trio at the Max Planck Institute for Human Cognitive and Brain Sciences, Leipzig, Germany. The DTI image is given as second order tensors at a resolution of $1.72 \mathrm{~mm}$. We used the tensorlines method introduced by Weinstein et al. [29][12] as implemented in MedINRIA [6] for generating all tracts used in this paper. To obtain a full brain tractogram, the tensorlines were seeded at voxels where the fractional anisotropy exceeds a certain threshold. However, other seeding strategies are possible as the used strategy does not imply any constraints on the design and implementation of the parts of the interactive visualization and analysis loop. The reconstructed tracts are stored into a file from which they can be loaded for the following steps as needed. The complete tractogram in our example consists of 74313 polylines represented by 5397993 vertices.

\subsection{EEG Source Reconstruction}

Electroencephalography (EEG) is a technique measuring the accumulated electrical activity inside the brain by recording voltage changes at the head surface. Compared to other brain measurement techniques EEG is recorded at relatively few (21 - 256) locations [19]. Although EEG is recorded from electrodes placed around the head surface and although the properties (amplitudes, frequencies, etc.) of the recorded voltage curves are expressive and meaningful themselves, one is interested in local currents inside the head because they are indicative for neuronal activity at the different locations in the brain. The methodology applied to obtain these currents is called source reconstruction. Considering, that the density of the source current at a 
certain location is a vector with three components, the problem of source reconstruction for more than 42 locations from, e.g., 128 electrodes, is under-determined. This fact is a first hint at the comparatively low spatial accuracy of reconstructed sources. To get a higher accuracy, further constraints obtained from prior knowledge of the brain structure have to be imposed [19]. Still, the achievable spatial resolution is much worse than that of MRI or CT. Despite these disadvantages, EEG is an important tool for examining brain activity and function because of its high resolution in time. Together with the often simultaneously discussed magnetoencephalography (MEG), EEG is the only common measurement technique that is able to capture the fast current changes in the brain, which can occur within milliseconds.

In this paper, we use the ASA software package [31] for source reconstruction and dipole localization. ASA (Advanced Source Analysis) is a highly flexible package for analysis of continuous and event-related EEG/MEG signals. Regarding our task, it provides a variety of useful source reconstruction and signal analysis features. For single dipoles a spatio-temporal dipole fit as described by Scherg [19] is used. A tutorial for using ASA for source reconstruction can be found here: [1].

Like the tracts, the EEG and the reconstruction results are stored into files from which they can be loaded for the following steps as needed.

\section{Technique}

After preprocessing has been performed, the data are ready for exploration using the technique proposed in this paper (see Fig. 3). Our specific approach is a combination of visualization and interaction techniques. In the following subsection, we first describe the visualization of the fibers and of the EEG on the head surface. Afterwards, we describe how we present the original EEG signal and how this presentation is used for interactively navigating through the time-dependent data.

\subsection{Visualization}

The fibers are visualized using the techniques available in OpenWalnut [5]. These include simple lines (Fig. 2), illuminated lines (Fig. 1), and fake tubes (Fig. 4). Each of these line types can be rendered with a user-defined constant color as well as with either local or global directional color coding.

Rendering only a subset of a set of fibers, e.g. of a whole brain tractogram, can be achieved by defining regions of interest (ROIs). As a result only those fibers which run through the defined ROIs will be shown. This selection mechanism is an implementation of the approach reported by Blaas et al. [3]. As the selection mechanism allows the combination of different ROIs for selecting a single fiber bundle, it is also possible to select those fibers that run through the locations of 
several simultaneously active dipoles. Furthermore, multiple bundles each running through one of the ROIs can be selected. An example of the latter is shown in Fig. 4.

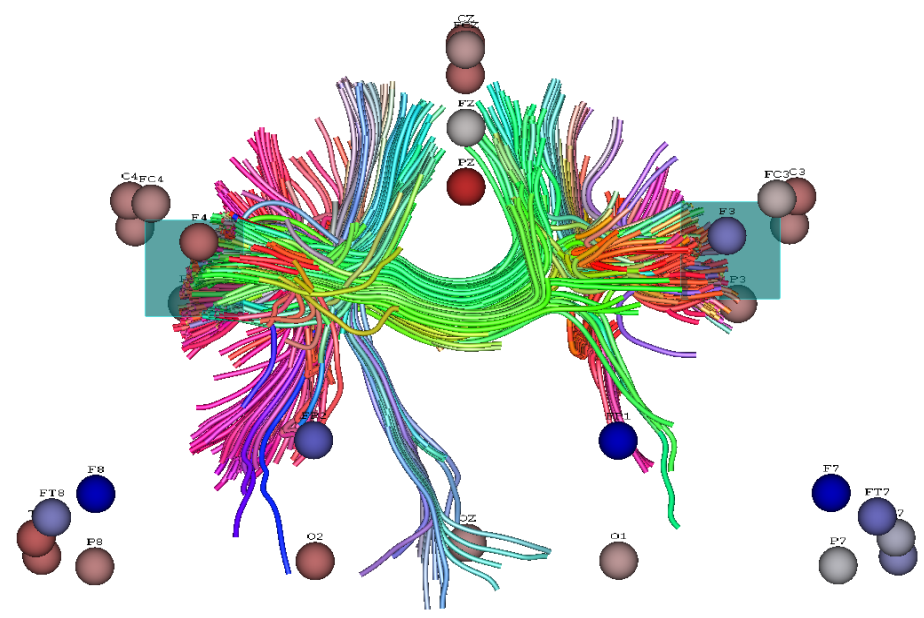

Fig. 4 Two ROI boxes (20mm) selecting fibers are the result of two simultaneously active dipoles $(\mathrm{t}=128.433)$.

The presentation of the original EEG signal is described in Sec. 3.2 because this presentation is also used for navigation in time.

In order to give a quick overview of the magnitude distribution at the currently selected point in time, we color-code the magnitudes of the different channels on the head surface (see Fig. 2). In our case the "head surface" is a simple interpolation of the electrode positions (Delaunay triangulation). We use a 2D implementation of the triangulation algorithm. Therefore we have to project the positions to two dimensional space first. A projection along the axial direction yields satisfactory results most of the time. In some cases, however, where the electrode positions cover more than the upper hemisphere, i.e. where parts of the projection overlap, we have to spread the lower positions somewhat before performing the projection. This prevents overlapping triangles in the projection and thus guarantees a valid triangulation. To color the triangles connecting the electrode positions, we map values, that where linearly interpolated from the values at the electrodes, to color.

\subsection{Navigation}

The user interface for navigating through the EEG data along the time dimension is visible in the lower left widget in Fig. 2. It is realized as a kind of timeline widget [20]. As in most EEG software, the standard presentation shows the signals of the different electrodes (channels) as curves in row-oriented layout. The name of the 
electrode belonging to a certain row is always visible on the left. A vertical red line indicates the currently selected point in time. The position of this line and thus the selected time value can be adjusted by left-clicking in the widget. This very simple type of interaction makes data exploration in time feasible and intuitive for all (also new) users. As exploring the correspondence of the instantaneous EEG signal and the fibers selected for dipoles at the same instant of time are the main goal of this work, the simple time point selection is central to the interaction and navigation. Dragging with the middle mouse button allows to bring additional channels (moving up/down) or other time intervals (moving left/right) of the recording into view. This is important for exploring the signal captured at all electrodes and thus to get an impression of the complete momentary state of the EEG. Dragging (left/right) with the right mouse button zooms (out/in) the currently visible time interval. The mouse wheel adjusts the scaling of the curves, i.e. the mapping from the measured voltage to the height of the curve. The different zoom and scaling levels are useful for getting an oveverview or inspecting details of the EEG curves. All mentioned adjustable values (position, scaling, etc.) can also be adapted in the control panel (right in Fig. 2). This allows to select specific numeric values by typing the exact numbers and can be useful for quantitavive studies where the exact values are needed.

An alternative EEG representation, called butterfly plot, which superimposes the signals of all channels, is visible in Fig. 1. This representation highlights certain particularly interesting instants of time, while, due to visual clutter, the single curves are not discernible anymore. All interaction types described for the conventional representation are also available for the butterfly plot. As a result, navigation in time is possible in both representations by simply clicking at the position of the desired instant of time.

If immediate source reconstruction in the background was possible, we could simply determine active locations and thus ROIs (see Sec. 3.3) for any selected instant of time. However, as source reconstruction is a time consuming process and as we have to rely on externally performed dipole localization, we only have dipoles for a number of time intervals. These time intervals are highlighted in the EEG plot window as shown in Fig. 2. A white background means no dipole; color indicates the presence and magnitude (by color saturation) of a loaded dipole.

When the user selects a certain point in time, the system checks if there are active dipoles at this time or in other words, if the selected instant of time lies in a time interval of one of the dipoles. The positions of the respective dipoles (one or multiple) are then used for fiber selection. The selection will be described in the Sec. 3.3 in more detail.

Scrolling through the whole EEG plot, searching for dipoles can be a tedious task for plots representing a long recording session. Therefore, we introduced another navigation mode, which we call snap to dipole (see Fig. 2, "Settings" tab). This mode is tailored to support fast selection of times for which dipoles have been loaded. If this mode is active and the user clicks, i.e. selects a certain point in time, the time marker snaps to the next position in time where a dipole can be found. One can think of this as a nearest neighbor search. The position located by the snapping will be the border of an interval containing an active dipole. The dipole magnitude 


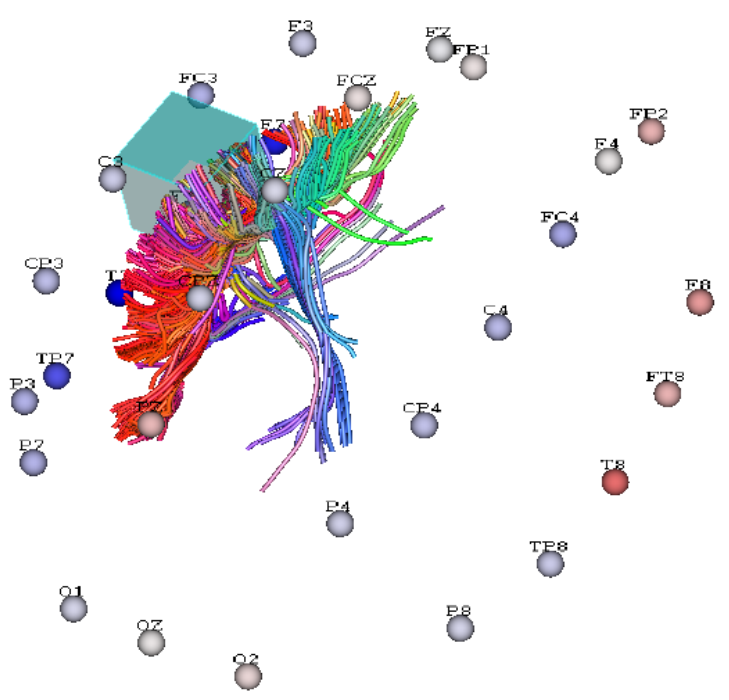

Fig. 5 Fiber selection resulting from ROI box with $3 \mathrm{~mm}$ edge length located at reconstructed dipole position $(\mathrm{t}=117.889)$.

will thus be relatively low at this point. With the color coding, however, it is now easy to navigate to positions at which the magnitude is higher. The snapping is especially important because the active dipoles often cover only a small portion of the whole time of the loaded EEG data.

\subsection{Combination}

As alluded to before, the combined visualization or combined exploration of fiber and EEG data is achieved by generating regions of interest (ROIs), in our case boxes, representing the dipoles active at the currently selected point in time.

The generated ROI box is centered at the given dipole position. As a first approximation we use a box with $30 \mathrm{~mm} \times 30 \mathrm{~mm} \times 30 \mathrm{~mm}$ extent. A selection exemplifying the result of such a ROI is shown in Fig. 5. The initial box size is a conservative choice in the sense that it selects relatively many fibers instead of showing too few and thus missing the important ones. See Scherg [19] for typical localization errors that led to our choice of the box size. The user can decrease the size of the ROI to explore fibers that would be of interest if the activation was smaller or more accurately localizable than the initial region. When changing its size, the ROI will grow or shrink equally in all directions and will thus stay centered at the dipole position.

As described in Sec. 3.1, the fiber selection mechanism is very flexible in combining the effects of different ROIs. We employ this for dealing with the case of 
multiple simultaneously active dipoles. The usual use case is to show all fibers that are related to any of the dipoles. This is demonstrated in Fig. 4 for two dipoles. A second option is to select only those fibers that run through all ROIs at the same time. However, one should be aware that this can results in showing no or only very few fibers for some dipole configurations. Even in the examples shown in Fig. 4, there is no fiber that runs through both ROIs. Actually, only one fiber of the corpus callosum is selected by the right ROI and this fiber does not pass through the ROI in the left part of the image. Fig. 5 shows that even increasing the ROI size results in the selection of only two fibers of the corpus callosum.

\section{Implementation Details}

Most data stemming from measuring, e.g. imaging, the brain is small enough to easily fit into the main memory of current commodity hardware. EEG data, in contrast, can become very large due to the number of electrodes used and the time recorded. In principal, increasing recording time can make the data arbitrarily large.

Handling such data makes it necessary to keep the treated file open and read only the data that are currently needed into main memory. The direct access to the file is realized by a pager (WPagerEEG, Fig. 6) which can be implemented for different file formats. The currently supported format is CNT (continuous EEG data file). For accessing such files we use the open source library Libeep [22]. As the metadata contained in the CNT file, like information about the measurement channels, is comparatively small, we load all metadata in the beginning. Only the voltage data, i.e. the data measured for each channel, is loaded on demand by the pager. Modules that need access to the voltage data ask the EEG dataset (WEEG2) for it by providing a time interval. The dataset class in turn requests the data from the pager. The pager then loads the requested data from the file if it is not yet present in memory. It presents the data to the dataset and removes unused data from memory.

More details of the implementation (Fig. 6) are described in a master's thesis [17] and the source code of our implementation is available in the online repository of OpenWalnut [5].

\section{Conclusion}

The presented system and techniques allow for a new type of visualization and exploration of DTI fiber pathways in conjunction with EEG and MEG source reconstruction data. The combination of the two modalities provides a platform for deeper investigations of the relation between anatomical structures, especially connections, in the brain and certain cognitive functions that these structures are involved in. An intuitive interaction mechanism, specially tailored to the characteristics of the com- 


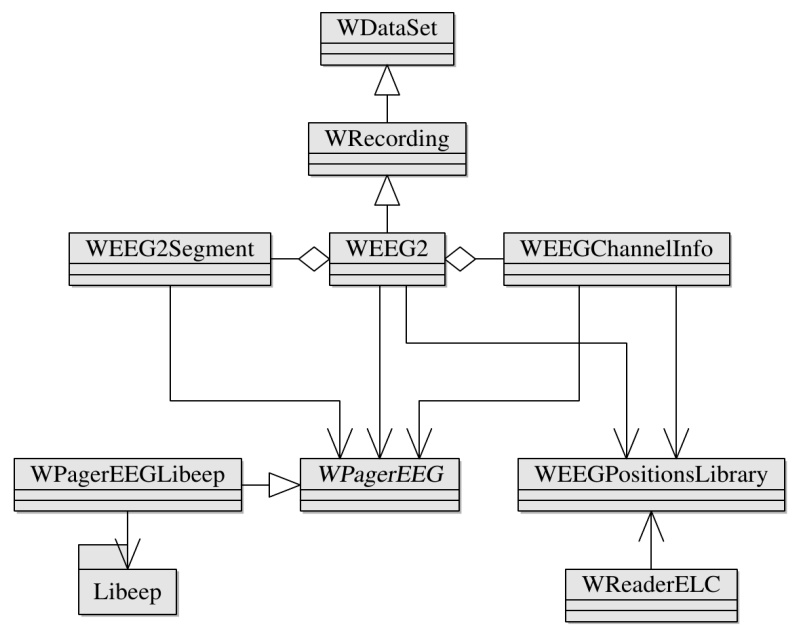

Fig. 6 Data handling scheme.

bination of EEG and fiber pathways, has been introduced. This mechanism is the heart of the presented system as it enables the user driven exploratory process.

All presented techniques are freely available in the open source visualization system OpenWalnut (http: / / www . openwa l nut . org). The data can be loaded using the general data import mechanism in combination with the Read Dipoles module. The visualization as well as the interaction techniques presented in this paper are integrated in the EEG View module.

One of our future research directions will be the relatively straightforward extension of the framework to MEG instead of EEG data. Further possible directions are the combination of the presented fiber bundle selection mechanism with bundle visualization techniques already available in OpenWalnut, e. g. bundle parameter and bundle surface visualization [7], and employing advanced ROI definitions, e.g. on the basis of distributed sources [14], deviating from cubes.

\section{Acknowledgments}

We thank the OpenWalnut community for providing their software as basis for implementing the presented techniques. We are also grateful to Gerik Scheuermann and his group at the University of Leipzig, who provided an enjoyable and inspiring environment for Cornelius Müller's work on his master's thesis. The reviewers made many very valuable suggestions, we would like to thank them for this. This work was partly supported by the AiF (ZIM grant KF 2034701SS8). 


\section{References}

1. ANT BV, Enschede, Netherlands. ASA Getting Started, September 2009.

2. J. I. Berman, S. Chung, P. Mukherjee, C. P. Hess, E. T. Han, and R. G. Henry. Probabilistic streamline q-ball tractography using the residual bootstrap. NeuroImage, 39(1):215-222, 2008.

3. J. Blaas, C. P. Botha, B. Peters, F. M. Vos, and F. H. Post. Fast and reproducible fiber bundle selection in DTI visualization. In C. Silva, E. Gröller, and H. Rushmeier, editors, Proceedings of IEEE Visualization 2005, pages 59-64, October 2005.

4. S. S. Dalal, J. M. Zumer, A. G. Guggisberg, M. Trumpis, D. D. E. Wong, K. Sekihara, and S. S. Nagarajan. MEG/EEG source reconstruction, statistical evaluation, and visualization with NUTMEG. Computational Intelligence and Neuroscience, 2011, 2011.

5. S. Eichelbaum, M. Hlawitschka, A. Wiebel, and G. Scheuermann. OpenWalnut - an opensource visualization system. In W. Benger, A. Gerndt, S. Su, W. Schoor, M. Koppitz, W. Kapferer, H.-P. Bischof, and M. D. Pierro, editors, Proceedings of 6th High-End Visualization Workshop, pages 76-78, Dec. 2010.

6. P. Fillard, N. Toussaint, and X. Pennec. MedINRIA: DT-MRI processing and visualization software, November 2006. Guest paper at the Similar Tensor Workshop, Las Palmas, Spain.

7. M. Goldau, A. Wiebel, M. Hlawitschka, G. Scheuermann, and M. Tittgemeyer. Visualizing DTI parameters on boundary surfaces of white matter fiber bundles. In J. Zhang, editor, Proceedings of the Twelfth IASTED International Conference on Computer Graphics and Imaging, pages 53-61. International Association of Science and Technology for Development, ACTA Press, February 2011.

8. S. H. Jang, S. H. Ahn, J. S. Ha, S. J. Lee, J. Lee, and Y. H. Ahn. Peri-infarct reorganization in a patient with corona radiata infarct: A combined study of functional MRI and diffusion tensor image tractography. Restorative Neurology and Neuroscience, 24(2):65-68, Jan 2006.

9. M. Jenkinson, C. Beckmann, T. Behrens, M. Woolrich, and S. Smith. Fsl. Neurolmage, 62(2):782-790, August 2012.

10. W.-K. Jeong, P. T. Fletcher, R. Tao, and R. Whitaker. Interactive visualization of volumetric white matter connectivity in DT-MRI using a parallel-hardware Hamilton-Jacobi solver. IEEE Transactions on Visualization and Computer Graphics, 13(6):1480-1487, 2007.

11. C. R. Johnson, R. MacLeod, S. G. Parker, and D. Weinstein. Biomedical computing and visualization software environments. Communications of the ACM, 47(11):64-71, Nov. 2004.

12. G. Kindlmann. Visualization and Analysis of Diffusion Tensor Fields. PhD thesis, School of Computing, University of Utah, September 2004.

13. T. R. Knösche. Solutions of the neuroelectromagnetic inverse problem - an evaluation study. PhD thesis, Universiteit Twente, 1997.

14. T. R. Knösche, P. Praamstra, D. Stegeman, and M. Peters. Linear estimation discriminates midline sources and a motor cortex contribution to the readiness potential. Electroencephalography and Clinical Neurophysiology, 99(2):183 - 190, 1996.

15. M. J. Lowe, C. Horenstein, J. G. Hirsch, R. A. Marrie, L. Stone, P. K. Bhattacharyya, A. Gass, and M. D. Phillips. Functional pathway-defined MRI diffusion measures reveal increased transverse diffusivity of water in multiple sclerosis. NeuroImage, 32(3):1127 - 1133, 2006.

16. S. Mori and P. C. M. van Zijl. Fiber Tracking: Principles and Strategies - A Technical Review. NMR in Biomed., 15(7-8):468-480, 2002.

17. C. Müller. Kombinierte Visualisierung von EEG- und Diffusions-MRT-Nervenfaser-Daten. Master's thesis, Fakultät für Mathematik und Informatik, Universität Leipzig, April 2010.

18. M. Reisert, I. Mader, C. Anastasopoulos, M. Weigel, S. Schnell, and V. Kiselev. Global fiber reconstruction becomes practical. NeuroImage, 54(2):955 - 962, 2011.

19. M. Scherg. Functional imaging and localization of electromagnetic brain activity. Brain Topography, 5(2):103-111, 1992.

20. V. A. Schmidt. User interface design patterns. Technical report, DTIC Document, 2010.

21. T. Schonberg, P. Pianka, T. Hendler, O. Pasternak, and Y. Assaf. Characterization of displaced white matter by brain tumors using combined DTI and fMRI. NeuroImage, 30(4):1100 1111, 2006. 
22. R. Smies. Libeep. http://sourceforge. net/projects/libeep/. Last accessed 2012-04-13.

23. O. Sourina, A. Sourin, and V. Kulish. EEG data driven animation and its application. In A. Gagalowicz and W. Philips, editors, Computer Vision/Computer Graphics Collaboration Techniques, volume 5496 of Lecture Notes in Computer Science, pages 380-388. Springer Berlin / Heidelberg, 2009.

24. F. Tadel, S. Baillet, J. C. Mosher, D. Pantazis, and R. M. Leahy. Brainstorm: A user-friendly application for MEG/EEG analysis. Computational Intelligence and Neuroscience, 2011, 2011. Article ID 879716,

25. M. ten Caat. Multichannel EEG Visualization. PhD thesis, Institute of Mathematics and Computing Science, University of Groningen, The Netherlands, February 12008.

26. N. Toussaint, J. C. Souplet, and P. Fillard. MedINRIA: Medical Image Navigation and Research Tool by INRIA. In Proc. of MICCAI'07 Workshop on Interaction in medical image analysis and visualization, Brisbane, Australia, 2007.

27. M. Wagner and M. Fuchs. Integration of functional MRI, structural MRI, EEG, and MEG. International Journal of Bioelectromagnetism, 3(1), March 2001.

28. B. Weiland, J. Moran, S. Bowyer, B. Smith, G. Barkley, and N. Tepley. DTI fiber tracts connect epileptic networks imaged with MEG coherence analysis. In 16th International Conference on Biomagnetism, Sapporo JAPAN, August 2008. Japan Biomagnetism and Bioelectromagnetics Society.

29. D. Weinstein, G. Kindlmann, and E. Lundberg. Tensorlines: Advection-diffusion based propagation through diffusion tensor fields. In Proceedings of IEEE Visualization '99, pages 249253, 1999.

30. C. H. Wolters, A. Anwander, X. Tricoche, S. Lew, and C. R. Johnson. Influence of local and remote white matter conductivity anisotropy for a thalamic source on EEG/MEG field and return current computation. International Journal of Bioelectromagnetism, 7, 2005.

31. F. Zanow and T. Knösche. ASA - Advanced source analysis of continuous and event-related EEG/MEG signals. Brain Topography, 16(4):287-290, 2004. 